\title{
Diaphragm weakness in mechanically ventilated critically ill patients
}

\author{
Gerald S Supinski ${ }^{*}$ and Leigh Ann Callahan ${ }^{\dagger}$
}

\begin{abstract}
Introduction: Studies indicate that mechanically ventilated patients develop significant diaphragm muscle weakness, but the etiology of weakness and its clinical impact remain incompletely understood. We assessed diaphragm strength in mechanically ventilated medical ICU patients, correlated the development of diaphragm weakness with multiple clinical parameters, and examined the relationship between the level of diaphragm weakness and patient outcomes.
\end{abstract}

Methods: Transdiaphragmatic twitch pressure (PdiTw) in response to bilateral magnetic stimulation of the phrenic nerves was measured. Diaphragm weakness was correlated with the presence of infection, blood urea nitrogen, albumin, and glucose levels. The relationship of diaphragm strength to patient outcomes, including mortality and the duration of mechanical ventilation for successfully weaned patients, was also assessed.

Results: We found that infection is a major risk factor for diaphragm weakness in mechanically ventilated medical ICU patients. Outcomes for patients with severe diaphragm weakness (PdiTw $<10 \mathrm{cmH}_{2} \mathrm{O}$ ) were poor, with a markedly increased mortality (49\%) compared to patients with PdiTw $\geq 10 \mathrm{cmH}_{2} \mathrm{O}(7 \%$ mortality, $P=0.022)$. In addition, survivors with PdiTw $<10 \mathrm{cmH}_{2} \mathrm{O}$ required a significantly longer duration of mechanical ventilation $(12.3 \pm$ 1.7 days) than those with $\mathrm{PdiTw} \geq 10 \mathrm{cmH}_{2} \mathrm{O}(5.5 \pm 2.0$ days, $P=0.016)$.

Conclusions: Infection is a major cause of severe diaphragm weakness in mechanically ventilated patients. Moreover, diaphragm weakness is an important determinant of poor outcomes in this patient population.

\section{Introduction}

The number of mechanically ventilated patients in medical ICUs (MICUs) in the United States has increased dramatically over the past 20 years. Currently 800,000 patients per year require mechanical ventilation [1]. Many of these patients die, with an annual mortality exceeding 200,000 [2]. In addition, survivors often require prolonged, expensive hospital stays to achieve liberation from mechanical ventilation [3]. In the past it was thought that the severity of lung disease was the major determinant of outcomes in MICU patients, but recent work indicates that mechanically ventilated patients develop significant diaphragm weakness [4-6].

Diaphragm weakness is primarily thought to occur as a consequence of ventilator-induced diaphragm inactivity,

\footnotetext{
* Correspondence: gsupi2@email.uky.edu

† Contributed equally

Department of Internal Medicine, Division of Pulmonary, Critical Care and Sleep Medicine, University of Kentucky, 740 South Limestone Room L-543, Lexington, KY 40536-0284, USA
}

(c) 2013 Supinski and Callahan; licensee BioMed Central Ltd. This is an open access article distributed under the terms of the Creative

with weakness progressing as duration of mechanical ventilation increases $[7,8]$. Theoretically, however, there are other mechanisms by which diaphragm weakness can develop. Animal studies indicate that experimental models of infection induce significant diaphragm weakness $[9,10]$. In addition, data suggest that azotemia, hyperglycemia, and low systemic albumin levels are risk factors for prolonged mechanical ventilation and could theoretically be associated with the development of respiratory muscle weakness [11-13]. The importance of infection, azotemia, hyperglycemia, and reduced albumin levels as risk factors for the development of diaphragm weakness in mechanically ventilated patients, however, is unknown.

Diaphragm weakness is also commonly thought to predispose patients to sustained respiratory failure, greatly prolonging the time required to wean patients from mechanical ventilation and worsening clinical outcomes. No previous study, however, has examined the quantitative relationship of diaphragm function, assessed using a 
purely objective, nonvolitional technique (such as bilateral anterior magnetic phrenic nerve stimulation), to clinical outcomes in mechanically ventilated patients.

The purpose of the present study, therefore, was to objectively measure diaphragm strength in a crosssection of mechanically ventilated MICU patients and to test the specific hypothesis that the severity of diaphragm weakness would correlate with one or more of the following clinical factors: the presence of infection, blood urea nitrogen level, serum albumin level, and/or blood glucose level. We also ascertained the relationship of diaphragm strength to patient outcomes, including mortality, rate of transfer to long-term acute care (LTAC) facilities, and the subsequent duration of mechanical ventilation in MICU survivors who were successfully extubated. Finally, to determine whether clinicians were cognizant of the severity of diaphragm weakness present in their patients, we asked the attending MICU physicians to estimate diaphragm strength and compared these estimates with objectively determined measurements.

\section{Methods}

\section{Study protocol}

Studies were performed on adult ICU patients requiring mechanical ventilation in the University of Kentucky MICU for more than 24 hours. The protocol was approved by the University of Kentucky Institutional Review Board and informed consent was obtained from subjects and their surrogates. The following were recorded: diaphragm strength by measuring the transdiaphragmatic twitch pressure (PdiTw); respiratory static system compliance and airway resistance using the mechanical ventilator diagnostic module; basic clinical data; clinician estimates of diaphragm strength; and outcomes, including mortality, rate of transfer to LTAC facilities, and additional days required for continued mechanical ventilation until successful extubation.

\section{Exclusion criteria}

If the attending physician anticipated that the patient would be successfully weaned from mechanical ventilation in less than 24 hours, or determined that the patient was too unstable to tolerate the measurements, subjects were not screened for study inclusion. Exclusion criteria included: requirement for high dose pressors $(\geq 15 \mu \mathrm{g} /$ minute norepinephrine or $\geq 15 \mathrm{mg} / \mathrm{kg} /$ minute dopamine); elevated positive end-expiratory pressure (PEEP $\geq 15 \mathrm{cmH}_{2} \mathrm{O}$ ); presence of a cardiac pacemaker or implanted defibrillator; administration of neuromuscular blocking agents within 48 hours prior to study entry; recent variceal bleeding; pregnancy; incarceration; or institutionalization.

\section{Determination of transdiaphragmatic twitch pressure}

Diaphragm strength was assessed by measuring PdiTw in response to bilateral anterior magnetic stimulation of the phrenic nerves. PdiTw is an objective, nonvolitional technique that has been verified in previous studies to provide the most accurate assessment of diaphragm strength in humans [14-16]. Moreover, previous studies demonstrate that this technique can reliably and reproducibly measure diaphragm contractile strength in mechanically ventilated ICU patients [4-6].

Subjects were studied in the supine position with the head of the bed elevated at $30^{\circ}$. Two sterile commercially available balloon-tipped catheters (Ackrad Laboratories, NJ, USA) were passed through the nose after application of local anesthetic (1 milliliter of $1 \%$ Lidocaine gel); one catheter was placed in the stomach, while the other was placed in the esophagus. Following initial placement, the catheters were connected to Validyne pressure transducers (Validyne Engineering, Northridge, CA, USA) to verify correct positioning. Correct placement of the gastric balloon was confirmed by demonstrating a positive pressure in response to pressure applied over the stomach; correct placement of the esophageal balloon was verified by demonstrating that the pressure waveform had an end-expiratory pressure similar to the total PEEP level and also mirrored airway pressure changes with inspiratory efforts during airway occlusion.

After confirming accurate balloon placement, subjects were left to breathe quietly for 10 minutes before further assessment. Figure of eight magnetic coils attached to dual Magstim 200 stimulators (Jali Medical, Inc., Waltham, MA, USA) were then placed bilaterally over the phrenic nerves adjacent to the border of the sternocleidomastoid muscles. Magnetic field strength was adjusted to maximal levels (100\%) and simultaneous supramaximal magnetic pulses were delivered to the phrenic nerves bilaterally to elicit maximal twitch transdiaphragmatic pressures (that is, PdiTw). Stimuli were interpolated between adjacent ventilator breaths and the transdiaphragmatic pressures elicited by these stimuli were recorded while simultaneously and transiently occluding the external circuit connecting the endotracheal tube to the ventilator with a pneumatic valve. A minimum of five twitches were recorded, with at least 30 seconds between adjacent stimuli. To verify stimuli were supramaximal, additional twitches were performed at reduced magnetic field strengths (90 to 95\%). PdiTw was calculated as follows:

$$
\text { pdiTw }=\Delta \text { gastric pressure }-\Delta \text { esophageal pressure }
$$

The best three measurements in response to $100 \%$ levels of magnetic stimulation were averaged for each subject and recorded as the PdiTw. 


\section{Measurement of respiratory system static compliance and airway resistance}

For these assessments, the ventilator was set to a square-wave flow pattern with an inspiratory plateau. The ventilator rate was then transiently increased (for example, 30 to 60 seconds) to suppress spontaneous respirations. After reaching a steady state, the peak pressure and the plateau pressure were recorded and intrinsic PEEP was determined using an end-expiratory occlusion maneuver. Inspiratory airway resistance of the respiratory system was calculated as:

\section{(Peak pressure - plateau pressure)/inspiratory flow}

The effective static compliance of the respiratory system was calculated as:

$$
\text { Tidal volume/(plateau pressure - (total PEEP)) }
$$

Once measurements were completed, the ventilator was returned to its previous mode and settings.

\section{Clinical parameters}

Data for the following clinical parameters were collected as close as possible to the time of determination of PdiTw levels: age, gender, clinical diagnoses, the presence of positive cultures for infectious agents, antibiotic regimen, glucose, albumin, blood urea nitrogen (BUN), Sequential Organ Failure Assessment scores, Charlson comorbidity indexes, vital signs, duration of mechanical ventilation prior to PdiTw measurement, mechanical ventilation mode, $\mathrm{FiO}_{2}$ (fraction of inspired oxygen), tidal volume and rate, percentage of patient triggered breaths, and most recent arterial blood gas values. All recorded values were obtained within 24 hours of PdiTw assessment.

\section{Clinician estimates}

Attending physicians were asked to estimate the level of diaphragm strength using a form with qualitative descriptors of muscle weakness (see Additional file 1).

\section{Statistical analysis}

Whenever data were normally distributed and variances were similar, parametric tests were used to compare groups. When these conditions were not met, nonparametric tests were used to make comparisons. Data analyzed using parametric tests are presented as mean \pm standard error of the mean. Data analyzed using nonparametric tests are presented as median \pm confidence intervals. Linear regression was utilized to assess the relationship of BUN, albumin, glucose and duration of prior mechanical ventilation to PdiTw level. Analysis of variance was employed to compare PdiTw across cohorts of patients with different levels of ventilator triggering. Fisher exact testing and receiver operating curve analyses were used to determine the boundary between weak and strong PdiTw groups that best discriminated between survival and mortality [17].

\section{Results}

\section{Diaphragm strength in medical ICU patients}

Sixty subjects were recruited into the study. PdiTw could not be measured in three subjects because the magnetic coils could not be effectively positioned due to anatomic constraints (Subjects 18, 43, and 48). Detailed information for the 57 subjects in whom PdiTw measurements were successfully performed is provided in Additional file 2 . To verify that we achieved supramaximal levels of magnetic stimulation, we plotted the PdiTw values achieved using 95\% magnetic field strength levels against PdiTw values attained using $100 \%$ magnetic field strength, as shown in Figure 1A. The PdiTw levels obtained using 95\% and 100\% field strength levels were virtually identical, arguing that supramaximal neural stimulation was achieved when employing 100\% magnetic field strength for these studies. Moreover, the twitch determinations were highly reproducible in individual subjects, with a coefficient of variation for the best three measurements performed at $100 \%$ stimulator output averaging $7 \%$ for the 57 subjects. High levels of PEEP can alter the relationship between the actual intrinsic diaphragm strength and the measured PdiTw. In the present cohort of patients, however, only one study subject had PEEP $>8 \mathrm{cmH}_{2} \mathrm{O}$. As a result, PEEP-induced hyperinflation did not appreciably impact our data analysis (see Additional file 3).

This cohort of 57 mechanically ventilated subjects had a mean PdiTw of $7.9 \pm 0.6 \mathrm{cmH}_{2} \mathrm{O}$. This value is similar to values reported previously in mechanically ventilated critically ill patients [4-6]. For comparison, normal healthy adults average a PdiTw of $29.3 \pm 2.8 \mathrm{cmH}_{2} \mathrm{O}$ in our laboratory; this value is similar to that reported for healthy adults in the literature $[4,14]$. Estimates of diaphragm strength from the attending physicians were obtained for 51 subjects. Clinicians did not accurately predict the level of diaphragm strength of their patients (Figure 1B). In many cases, patients with profound levels of diaphragm weakness were thought to have normal strength. Strength was overestimated in 46 of 51 patients, was correctly estimated in five patients, and was never underestimated.

\section{Risk factors for the development of diaphragm weakness} Data were analyzed to determine which factors correlated with the level of diaphragm weakness in mechanically ventilated subjects. We found a strong relationship between the presence of infection and diaphragm weakness. In all, 41 subjects were classified as being infected based on a positive test for a pathogenic organism from a sterile site 


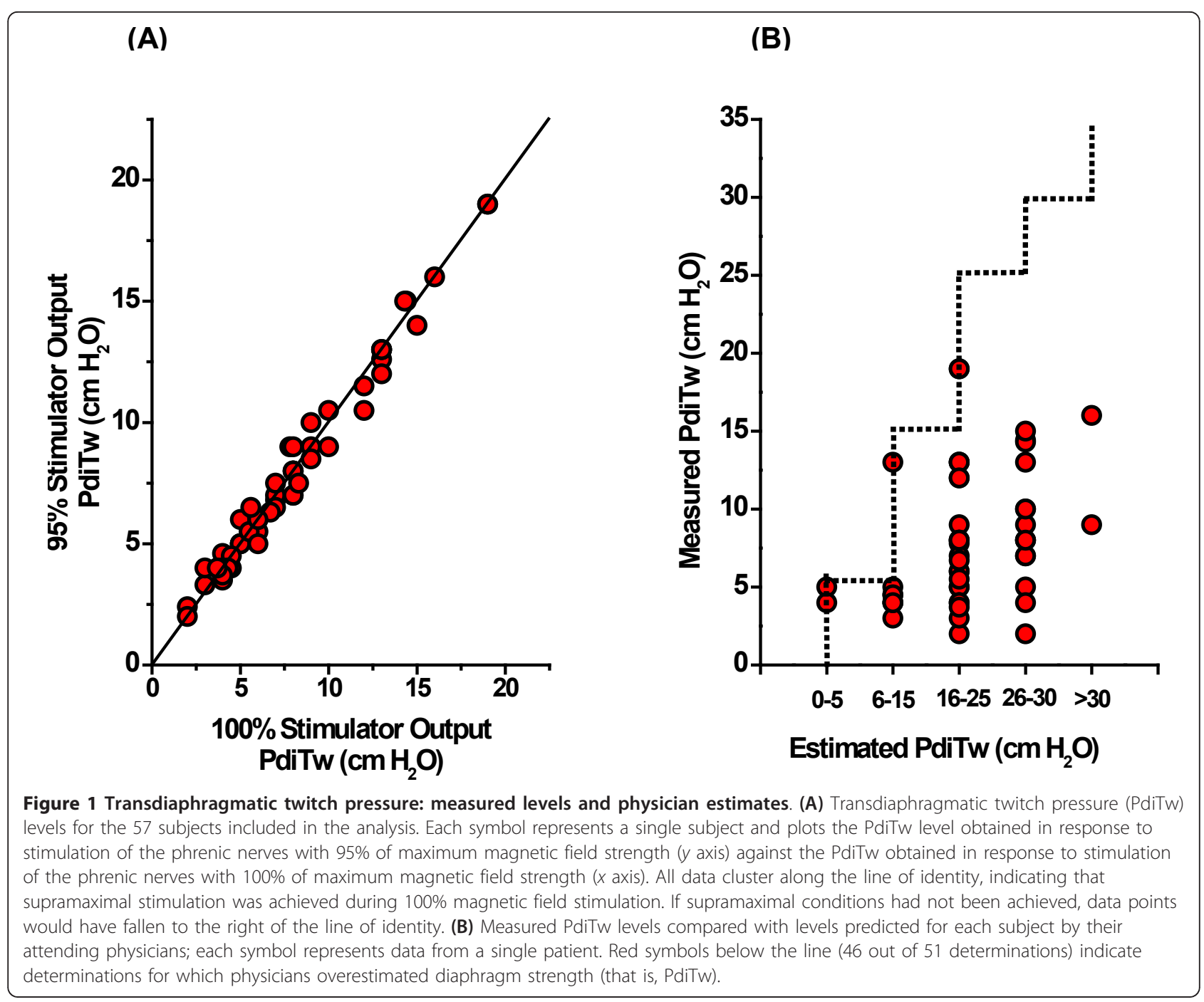

(40 patients) or a clinical diagnosis of bacterial pneumonia (one patient; cultures were lost for this individual). All 41 subjects classified as infected were thought by the attending physicians who were providing care for these patients to be infected clinically and all 41 of these patients received antibiotic therapy (Tables 2 and 3 ). The remaining 16 patients were classified as non-infected (see Table 2). Infected patients had a median PdiTw of only $5.5 \mathrm{cmH}_{2} \mathrm{O}$ (25 to $75 \%$ confidence levels of 4.0 to $7.9 \mathrm{cmH}_{2} \mathrm{O}$ ), while patients without clinical evidence of infection had a median PdiTw of $13.0 \mathrm{cmH}_{2} \mathrm{O}(25$ to $75 \%$ confidence levels of 11.0 to $14.7, P<0.001$ ) (Figure 2A). Of interest, while infection was associated with greater diaphragm weakness, infected patients did not have significantly different respiratory mechanical parameters (that is, respiratory system static compliance and airway resistance) from non-infected patients (Figure 2B, C).
We also found that there was no significant correlation between PdiTw and either BUN, albumin, or glucose levels (Figures 3A, B, C). While many patients were receiving steroids (regimens provided in Table 3 ), we found no correlation between steroid dosage and PdiTw values (see Additional file 4). In addition, we found no relationship between the number of days subjects had been on mechanical ventilation prior to testing and the level of PdiTw (Figure 4A). This finding contrasts with recent reports suggesting that patients on mechanical ventilation for longer durations have progressively lower levels of diaphragm strength $[6,18]$. One potential explanation for this difference is that the patients examined in the present study were all ventilated with assist modes of mechanical ventilation, while previous work that demonstrated a strong relationship between mechanical ventilation and the development of diaphragm weakness specifically restricted examination to patients who were on controlled 
Table 1 Characteristics of non-infected and infected study subjects

\begin{tabular}{lll}
\hline & $\begin{array}{l}\text { Non-infected } \\
(\boldsymbol{n}=\mathbf{1 6})\end{array}$ & $\begin{array}{l}\text { Infected } \\
(\boldsymbol{n}=\mathbf{4 1})\end{array}$ \\
\hline $\begin{array}{l}\text { Age (years) } \\
\text { Gender (\%) }\end{array}$ & $52.4 \pm 14.1$ & $55.5 \pm 16.7$ \\
$\quad$ Male & 44 & 49 \\
$\quad$ Female & 56 & 51 \\
Body mass index & $30.3 \pm 9.2$ & $29.8 \pm 9.5$ \\
Total ICU days & $15.1 \pm 9.8$ & $34.9 \pm 40.2$ \\
Days of MV before PdiTw & $9.1 \pm 8.9$ & $10.4 \pm 12.4$ \\
measurement & & 51 \\
Steroid usage (\%) & 50 & \\
\hline
\end{tabular}

Data presented as mean \pm standard deviation. $\mathrm{MV}$, mechanical ventilation; PdiTw, transdiaphragmatic twitch pressure.

mechanical ventilation with little or no spontaneous respiratory activity [18]. As shown in Figure 4B, our patient population had a high level of spontaneous respiratory activity, with the majority of patients triggering more than $75 \%$ of ventilator breaths. Of interest, we also found that PdiTw was similar over the range of levels of ventilator triggering observed in the present study (Figure 4C).

\section{Relationship of diaphragm strength to patient outcomes}

To assess the relationship between diaphragm strength and mortality, we plotted PdiTw against patient days of survival (Figure 5A). Patients that died were significantly weaker than survivors, with PdiTw averaging $6.3 \pm 0.6$ and $8.9 \pm 0.9 \mathrm{cmH}_{2} \mathrm{O}$, respectively, for these two groups $(P<0.04)$. To further analyze this relationship, we used Fisher exact testing and ROC curve analyses to determine the level of PdiTw that best discriminated between survival and mortality [17]. Both forms of testing found this boundary to be $10 \mathrm{cmH}_{2} \mathrm{O}$. Patients with PdiTw $\geq 10 \mathrm{cmH}_{2} \mathrm{O}$ had only a 7\% mortality (one death out of 14 patients) while patients with $\mathrm{PdiTw}<10 \mathrm{cmH}_{2} \mathrm{O}$ had a $49 \%$ mortality (17 deaths out of 35 patients, $P=0.022$ for comparison of the two groups; Figure $5 \mathrm{~B})$. Because indices of lung function may influence mortality, we also compared respiratory system static compliance and airway resistance between patients with PdiTw $\geq 10$ $\mathrm{cmH}_{2} \mathrm{O}$ and patients with Pdi $<10 \mathrm{cmH}_{2} \mathrm{O}$ (Figures 5C, D). Lung mechanics were not significantly different between these two groups of patients, indicating that level of diaphragm function, not lung function, best correlated with survival in our patients. In addition, patients with PdiTw $\geq 10 \mathrm{cmH}_{2} \mathrm{O}$ and PdiTw $<10 \mathrm{cmH}_{2} \mathrm{O}$ had similar Sequential Organ Failure Assessment scores (7.6 \pm 0.6 and $6.9 \pm 0.4$, respectively) and Charlson Comorbidity Indices ( $2.7 \pm 0.5$ and $2.5 \pm 0.3$, respectively).

We also evaluated the possible mechanism(s) by which diaphragm weakness may have influenced the incidence of death. In this cohort, five of the patients with PdiTw
$<10 \mathrm{cmH}_{2} \mathrm{O}$ that died were receiving vasopressors when care was withdrawn; vasopressors and mechanical ventilation were stopped simultaneously in these patients and death occurred as a result of combined respiratory failure and hypotension. In the remaining 12 patients with PdiTw $<10 \mathrm{cmH}_{2} \mathrm{O}$ that died, none met criteria for brain death, all maintained motor drive to the respiratory pump, none were on vasopressors, and the only form of continuous life support that these patients were receiving was mechanical ventilation. Prior weaning trials had been attempted and all 12 patients had failed to reach extubation criteria. Death occurred in these 12 patients when mechanical ventilation was withdrawn. These data suggest that the presence of severe diaphragm weakness limited weaning trial success in these 12 patients and may have influenced the decision to withdraw care.

With respect to other outcome measures, seven patients with PdiTw $<10 \mathrm{cmH}_{2} \mathrm{O}$ were transferred to a long-term ventilator facility while only one of the patients with PdiTw $\geq 10 \mathrm{cmH}_{2} \mathrm{O}$ was transferred to a LTAC facility. In addition, the time required to wean survivors from mechanical ventilation was a function of PdiTw, with time to wean increasing significantly for patients with PdiTw $<10 \mathrm{cmH}_{2} \mathrm{O}$ (Figure 6A). On average, duration of mechanical ventilation after PdiTw measurements was $12.3 \pm 1.7$ days for patients with PdiTw $<10 \mathrm{cmH}_{2} \mathrm{O}$ but only $5.5 \pm$ 2.0 days for patients with PdiTw $\geq 10 \mathrm{cmH}_{2} \mathrm{O}(P=0.016)$. In contrast, duration of mechanical ventilation had no relationship to either respiratory system static compliance (Figure 6B) or airway resistance (Figure 6C).

\section{Discussion}

The present study indicates that diaphragm weakness is a significant determinant for poor outcomes in mechanically ventilated MICU patients We found that the incidence of death was $49 \%$ in the patients with the weakest diaphragms (that is, with PdiTw $<10 \mathrm{cmH}_{2} \mathrm{O}$ ) but was only $7 \%$ for patients with PdiTw levels $\geq 10 \mathrm{cmH}_{2} \mathrm{O}$. One possible explanation for the far greater mortality in patients with PdiTw $<10 \mathrm{cmH}_{2} \mathrm{O}$ could be that weakness is simply a marker for multiorgan system failure and that damage to these other organs was primarily responsible for patient deaths. Surprisingly, however, we found that indices of disease severity (for example, lung mechanics, Sequential Organ Failure Assessment scores, Charlson Comorbidity Indexes) were almost identical in patients with PdiTw levels $\geq 10 \mathrm{cmH}_{2} \mathrm{O}$ and in patients with PdiTw $<10 \mathrm{cmH}_{2} \mathrm{O}$, suggesting that the relationship between diaphragm weakness and mortality is not simply an epiphenomenon. Moreover, the majority of patient deaths (12 of 18 deaths) were the direct result of withdrawal of mechanical ventilatory support in weak patients and, in each case, occurred after unsuccessful weaning 
Table 2 Classification of subjects according to presence/absence of active infection at time of PdiTw measurements ${ }^{\mathrm{a}}$

\begin{tabular}{|c|c|c|c|c|c|c|}
\hline $\begin{array}{l}\text { Subject } \\
\text { number }\end{array}$ & Infected & Site & Organism(s) isolated from site(s) & $\begin{array}{l}\text { Diagnosis of infection by } \\
\text { attending physician }\end{array}$ & $\begin{array}{l}\text { Antibiotic } \\
\text { therapy }^{\mathrm{b}}\end{array}$ & $\begin{array}{l}\text { Pulmonary } \\
\text { infiltrates }\end{array}$ \\
\hline 1 & No & & & No & No & No \\
\hline 2 & Yes & Liver abscess & $\begin{array}{l}\text { Staphylococcus sp., Fusobacterium } \\
\text { necrophorum }\end{array}$ & Yes & Yes & Yes \\
\hline 3 & No & & & No & No & No \\
\hline 4 & Yes & PAL & Streptococcus pneumonia & Yes & Yes & Yes \\
\hline 5 & No & & & No & No & No \\
\hline 6 & Yes & Blood & Gram-positive bacteriac ${ }^{c}$ & Yes & Yes & Yes \\
\hline 7 & Yes & PAL & Pseudomonas aeruginosa & Yes & Yes & Yes \\
\hline 8 & Yes & Blood & Staphylococcus species & Yes & Yes & Yes \\
\hline 9 & No & & & No & No & Yes $^{\mathrm{d}}$ \\
\hline 10 & Yes & PAL & Pseudomonas aeruginosa & Yes & Yes & Yes \\
\hline 11 & No & & & No & No & No \\
\hline \multirow[t]{2}{*}{12} & Yes & Sinuses & $\begin{array}{l}\text { Bacteroides capillosus, Fusobacterium } \\
\text { sp., } \beta \text {-strep. Gp C }\end{array}$ & Yes & Yes & Yes \\
\hline & & $\begin{array}{l}\text { Nasopharyngeal } \\
\text { swab }\end{array}$ & Influenza A & & & \\
\hline 13 & No & & & No & No & No \\
\hline 14 & Yes & Jejunal drain & VRE, Pseudomonas aeruginosa & Yes & Yes & Yes \\
\hline 15 & Yes & Blood & Staphylococcus aureus & Yes & Yes & No \\
\hline 16 & No & & & No & No & No \\
\hline \multirow[t]{2}{*}{17} & Yes & PAL & Staphylococcus species & Yes & Yes & Yes \\
\hline & & Blood & Staphylococcus species & & & \\
\hline 19 & Yes & PAL & Klebsiella pneumonia & Yes & Yes & Yes \\
\hline 20 & Yes & Blood & Staphylococcus aureus & Yes & Yes & No \\
\hline 21 & Yes & Neck abscess & Streptococcus sp. & Yes & Yes & No \\
\hline 22 & Yes & Sputum & Hemophilus parainfluenza & Yes & Yes & Yes \\
\hline \multirow[t]{2}{*}{23} & Yes & $\begin{array}{l}\text { Stage IV } \\
\text { decubitus }\end{array}$ & $\begin{array}{l}\text { Pseudomonas aeruginosa, Proteus } \\
\text { mirabilis }\end{array}$ & Yes & Yes & No \\
\hline & & Urine & Pseudomonas aeruginosa & & & \\
\hline 24 & No & & & No & No & No \\
\hline 25 & No & & & No & No & No \\
\hline 26 & No & & & No & No & No \\
\hline 27 & Yes & $\begin{array}{l}\text { Subphrenic } \\
\text { abscess }\end{array}$ & Candida glabrata & Yes & Yes & Yes \\
\hline 28 & No & & & No & No & No \\
\hline 29 & Yes & BAL & Escherichia coli, Klebsiella pneumoniae & Yes & Yes & Yes \\
\hline 30 & No & & & No & No & No \\
\hline 31 & No & & & No & No & Yes $^{d}$ \\
\hline 32 & Yes & Blood & Enterococcus faecalis & Yes & Yes & No \\
\hline 33 & Yes & PAL & Staphylococcus aureus & Yes & Yes & Yes \\
\hline 34 & Yes & Blood & Gram-positive cocci ${ }^{c}$ & Yes & Yes & Yes \\
\hline \multirow[t]{2}{*}{35} & Yes & ET aspirate & $\begin{array}{l}\text { Acinetobacter calcoaceticus, } \\
\text { Enterobacter cloacae }\end{array}$ & Yes & Yes & Yes \\
\hline & & Blood & Candida & & & \\
\hline \multirow[t]{2}{*}{36} & Yes & PAL & Achromobacter xyloxosidans & Yes & Yes & Yes \\
\hline & & $\begin{array}{l}\text { Central venous } \\
\text { catheter }\end{array}$ & Pseudomonas aeruginosa & & & \\
\hline 37 & Yes & PAL & Pseudomonas aeruginosa & Yes & Yes & Yes \\
\hline 38 & Yes & Liver abscess & Escherichia coli & Yes & Yes & Yes \\
\hline 39 & Yes & PAL & $\begin{array}{l}\text { Staphylococcus sp., Enterobacter } \\
\text { aerogenes, Escherichia coli }\end{array}$ & Yes & Yes & Yes \\
\hline \multirow[t]{2}{*}{40} & Yes & PAL & MRSA & Yes & Yes & Yes \\
\hline & & Blood & MRSA & & & \\
\hline
\end{tabular}


Table 2 Classification of subjects according to presence/absence of active infection at time of PdiTw measurements ${ }^{\mathrm{a}}$ (Continued)

\begin{tabular}{|c|c|c|c|c|c|c|}
\hline 41 & Yes & $\begin{array}{l}\text { Nasopharyngeal } \\
\text { swab }\end{array}$ & Influenza B & Yes & Yes & Yes \\
\hline \multirow[t]{3}{*}{42} & Yes & Blood & MRSA & Yes & Yes & Yes \\
\hline & & Leg abscess & MRSA & & & \\
\hline & & Osteomyelitis & MRSA & & & \\
\hline 44 & No & & & No & No & $Y_{e s}^{d}$ \\
\hline 45 & No & & & No & No & No \\
\hline 46 & Yes & Sputum & MRSA & Yes & Yes & Yes \\
\hline 47 & Yes & PAL & Streptococcus pneumonia & Yes & Yes & Yes \\
\hline 49 & No & & & No & No & No \\
\hline \multirow[t]{2}{*}{50} & Yes & Pleural fluid & Acinetobacter baumannii, VRE & Yes & Yes & Yes \\
\hline & & Pleural tissue & Acinetobacter baumannii, VRE & & & \\
\hline 51 & Yes & Blood & Bacillus circulans & Yes & Yes & Yes \\
\hline 52 & Yes & Blood & VRE & Yes & Yes & Yes \\
\hline \multirow[t]{2}{*}{53} & Yes & PAL & Pseudomonas aeruginosa & Yes & Yes & Yes \\
\hline & & $\begin{array}{l}\text { Nasopharyngeal } \\
\text { swab }\end{array}$ & H1N1 & & & \\
\hline \multirow[t]{2}{*}{54} & Yes & Blood & VRE & Yes & Yes & Yes \\
\hline & & Tracheal aspirate & Stenotrophomonas maltophilia & & & \\
\hline 55 & Yes & PAL & MRSA & Yes & Yes & Yes \\
\hline \multirow[t]{2}{*}{56} & Yes & Blood & MRSA, Pseudomonas aeruginosa, VRE & Yes & Yes & Yes \\
\hline & & Sputum & Pseudomonas aeruginosa & & & \\
\hline 57 & Yes & BAL & Samples lost ${ }^{\mathrm{e}}$ & Yes & Yes & Yes \\
\hline 58 & Yes & Tracheal aspirate & Hemophilus influenza & Yes & Yes & Yes \\
\hline 59 & Yes & Blood & Staphylococcus species & Yes & Yes & No \\
\hline \multirow[t]{2}{*}{60} & Yes & Urine & Enterobacter cloacae & Yes & Yes & Yes \\
\hline & & PAL & Streptococcus sp. & & & \\
\hline
\end{tabular}

${ }^{a}$ Data not included for Subjects 18,43 , and 48 because transdiaphragmatic twitch pressure (PdiTw) measurements were not obtained due to anatomic constraints. ${ }^{b}$ Patient on antibiotic therapy at the time of PdiTw measurement. ${ }^{\circ}$ Organism not speciated - subject treated with antibiotics prior to transfer. ${ }^{d}$ Patient with pulmonary infiltrates but without evidence of infection (Subject 9 with nonpulmonary acute lung injury, Subject 31 with pulmonary fibrosis, Subject 44 with

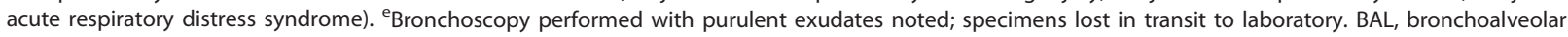
lavage; MRSA, methicillin-resistant Staphylococcus aureus; PAL, protected alveolar lavage; VRE, Enterococcus faecium (vancomycin-resistant).

Table 3 Medication regimen in 57 subjects at the time of transdiaphragmatic twitch pressure measurements ${ }^{a}$

\begin{tabular}{|c|c|c|c|}
\hline $\begin{array}{l}\text { Subject } \\
\text { number }\end{array}$ & Antibiotics & Other medications & Steroid regimen over entire ICU stay \\
\hline 1 & & $\begin{array}{l}\text { Midazolam, metoprolol, hydralazine, calcium acetate, } \\
\text { famotidine }\end{array}$ & \\
\hline 2 & $\begin{array}{l}\text { Piperacillin/tazobactam, } \\
\text { vancomycin, levofloxacin, } \\
\text { flagyl }\end{array}$ & Enoxaparin, omeprazole, dobutamine & Hydrocortisone 100 mg every 8 hours $\times 4$ days \\
\hline 3 & & Metoprolol, haloperidol, insulin, aspirin & \\
\hline 4 & $\begin{array}{l}\text { Piperacillin/tazobactam, } \\
\text { vancomycin, levofloxacin }\end{array}$ & Midazolam, fentanyl, pantoprazole & \\
\hline 5 & & Famotidine, trazadone & $\begin{array}{l}\text { Methylprednisolone } 1 \mathrm{~g} / \text { day } \times 3 \text { days, prednisone } \\
60 \mathrm{mg} / \text { day } \times 2 \text { days, } 40 \mathrm{mg} / \text { day } \times 3 \text { days, } 30 \mathrm{mg} / \text { day } \times \\
2 \text { days, } 20 \mathrm{mg} / \text { day } \times 2 \text { days, } 10 \mathrm{mg} / \text { day } \times 2 \text { days }\end{array}$ \\
\hline 6 & $\begin{array}{l}\text { Piperacillin/tazobactam, } \\
\text { vancomycin, fluconazole }\end{array}$ & $\begin{array}{l}\text { Midazolam, fentanyl, insulin, lactulose, levothyroxine, } \\
\text { norepinephrine, pantoprazole }\end{array}$ & Hydrocortisone 50 mg every 6 hours $\times 1$ day \\
\hline 7 & Piperacillin/tazobactam & $\begin{array}{l}\text { Midazolam, alprazolam, fentanyl, insulin, famotidine, } \\
\text { heparin }\end{array}$ & \\
\hline 8 & Vancomycin & $\begin{array}{l}\text { Midazolam, fentanyl, insulin, famotidine, heparin, } \\
\text { simvastatin, gabapentin, venlafaxine, calcium } \\
\text { gluconate }\end{array}$ & Hydrocortisone 50 mg every 8 hours $\times 8$ days \\
\hline
\end{tabular}


Table 3 Medication regimen in $\mathbf{5 7}$ subjects at the time of transdiaphragmatic twitch pressure measurements ${ }^{\mathrm{a}}$ (Continued)

9

Piperacillin/tazobactam, vancomycin

11

Vancomycin, clindamycin, tamiflu

Vancomycin, levofloxacin, flagyl, aztreonam

$$
\text { Vancomycin }
$$

\section{Vancomycin, levofloxacin}

Vancomycin, piperacillin/ tazobactam, levofloxacin

Vancomycin

Vancomycin, tobramycin, ampicillin/sulbactam

Vancomycin, levofloxacin

Vancomycin, doripenem, colistin

Doripenem, doxycycline, micafungin, flagyl

Doripenem, tobramycin, fluconazole, erythromycin aztreonam

Linezolid

\section{Vancomycin, piperacillin/} tazobactam, micofungin

Vancomycin, piperacillin/ tazobactam, micofungin, colistin, bactrim
Midazolam, lorazepam, omeprazole, ursodiol, clonidine, levetiracetam, ondansetron, metaclopramide, hydralazine, heparin

Midazolam, famotidine, aspirin, clopidogrel, insulin, heparin, metaclopramide, docusate

Midazolam, protonix, amitryptyline, bupropion, carvedilol, clonazepam, folic acid, acetaminophen, digoxin, ondansetron, heparin, tacrolimus

Midazolam, fentanyl, morphine, heparin, insulin, pantoprazole, bumetanide

Midazolam, enoxaparin, famotidine, insulin, metoprolol, aspirin, hydralazine, lisinopril, simvastatin

Midazolam, fentanyl, heparin

Propofol, omeprazole, heparin, amlodipine

Midazolam, fentanyl, protonix, ondansetron, darbepoetin, folic acid, cyanocobalamin, hydralazine, amldipine, lisinopril

Midazolam, fentanyl, protonix, insulin, lactulose, levothyroxine, sertraline, hydralazine, gabapentin, carvedilol

Heparin, hydralazine, labetalol, metoprolol, levetiracetam, lorazepam, omeprazole, phenytoin

Midazolam, morphine, amlodipine, labetalol, metoprolol, protonix, phenytoin, heparin

Midazolam, fentanyl, heparin, ibuprofen, amiodarone, metoprolol, omeprazole

Famotidine, heparin, metaclopramide, diphenhydramine

Bumetanide, midazolam, ascorbic acid, famotidine, insulin, vitamin A, zinc, aripiprazole, escitalopram, heparin

Paroxetine, pramipexole, simvastatin, omeprazole, heparin, midazolam, insulin, metoprolol, bumetanide Midazolam, heparin, omeprazole, haloperidol, olanzapine, insulin

Famotidine, darbepoetin, amlodipine, labetolol, folic acid, thiamine, dexmedetomidine, heparin

Midazolam, fentanyl, promethazine, pantoprazole, levetiracetam, propofol, lactulose

Midazolam, haloperidol, omeprazole, levothyroxine, heparin, insulin, furosemide

Morphine, ondansetron, rifaximin, pantoprazole, lactulose, insulin, aspirin, heparin, levetriacetam, midazolam, phenytoin, vasopressin

Aspirin, benztropine, famotidine, gabapentin, hydrochlorothiazide, metoclopramide, miralax, insulin, heparin, valproic acid, docusate

Azathioprine, bumetanide, clopidogrel, ezetimibe, famotidine, fentanyl, furosemide, heparin, metoprolol, midazolam, nitroglycerin patch, omeprazole, provastatin

Midazolam, fentanyl, aspirin, carbemazepam, haloperidol, metoprolol, omeprazole, phenytoin, insulin, heparin

Midazolam, lorazepam, fentanyl, aspirin, digoxin, insulin, metoprolol, famotidine, heparin

Midazolam, fentanyl, insulin, aspirin, amlodipine, metaclopramide, famotidine, heparin

Foscarnate, pantoprazole, diphenhydramine, insulin, fludrocortisone, levothyroxine, ursodiol
Prednisone $20 \mathrm{mg} /$ day $\times 3$ days, Hydrocortisone 100 mg every 8 hours $\times 2$ days

Methylprednisolone 60 mg every 12 hours $\times 3$ days

Methylprednisolone $60 \mathrm{mg} /$ day $\times 4$ days, prednisone $40 \mathrm{mg} /$ day $\times 3$ days

Methylprednisolone 60 mg every 12 hours $\times 6$ days

Prednisone $20 \mathrm{mg} /$ day $\times 2$ days

Prednisone $40 \mathrm{mg} /$ day $\times 6$ days

Prednisone $50 \mathrm{mg}$ every 8 hours $\times 8$ days

Methylprednisolone 125 mg every 6 hours $\times 2$ days

Methylprednisilone 125 mg every 6 hours $\times 3$ days,

$40 \mathrm{mg}$ every 6 hours $\times 2$ days, prednisone

$60 \mathrm{mg} /$ day $\times 5$ days

Methylprednisolone 60 mg every 6 hours $\times 6$ days

Methylprednisolone 100 mg every 12 hours $\times 9$ days

Methylprednisolone $10 \mathrm{mg} \times 1$ day 
Table 3 Medication regimen in $\mathbf{5 7}$ subjects at the time of transdiaphragmatic twitch pressure measurements ${ }^{\mathrm{a}}$ (Continued)

\begin{tabular}{|c|c|c|c|}
\hline 36 & $\begin{array}{l}\text { Vancomycin, } \\
\text { meraopenum, } \\
\text { valgancyclovir, cefipime, } \\
\text { dapsone, levofloxacin }\end{array}$ & $\begin{array}{l}\text { Ondansetron, omeprazole, metoprolol, } \\
\text { mycophenolate mofetil, sildenafil }\end{array}$ & Prednisone 25 mg/day $\times 250$ days \\
\hline 37 & $\begin{array}{l}\text { Piperacillin/tazobactam, } \\
\text { vancomycin, levofloxacin }\end{array}$ & $\begin{array}{l}\text { Midazolam, fentanyl, aspirin, heparin, omeprazole, } \\
\text { simvastatin }\end{array}$ & $\begin{array}{l}\text { Methylprednisolone } 40 \mathrm{mg} / \text { day } \times 2 \text { days, prednisone } \\
40 \mathrm{mg} / \text { day } \times 1 \text { day, prednisone } 20 \mathrm{mg} / \text { day } \times 3 \text { days }\end{array}$ \\
\hline 38 & $\begin{array}{l}\text { Flagyl, levofloxacin, } \\
\text { aztreonam }\end{array}$ & $\begin{array}{l}\text { Midazolam, fentanyl, morphine, norepinephrine, } \\
\text { vasopressin, famotidine, heparin }\end{array}$ & \\
\hline 39 & $\begin{array}{l}\text { Doripenam, colistimethate, } \\
\text { vancomycin }\end{array}$ & $\begin{array}{l}\text { Midazolam, fentanyl, lortab, zolpidem, metoprolol, } \\
\text { omeprazole, heparin }\end{array}$ & \\
\hline 40 & $\begin{array}{l}\text { Vancomycin, piperacillin/ } \\
\text { tazobactam, levofloxacin }\end{array}$ & $\begin{array}{l}\text { Midazolam, fentanyl, metaclopramide, aspirin, } \\
\text { azathioprine, clopidogrel, furosemide, ondansetron, } \\
\text { simvastatin, famotidine, heparin }\end{array}$ & Prednisone $20 \mathrm{mg} /$ day $\times 7$ days \\
\hline 41 & $\begin{array}{l}\text { Vancomycin, piperacillin/ } \\
\text { tazobactam, clindamycin }\end{array}$ & Midazolam, fentanyl, propofol, insulin, omeprazole & \\
\hline 42 & $\begin{array}{l}\text { Vancomycin, cefipime, } \\
\text { levofloxacin, acyclovir }\end{array}$ & $\begin{array}{l}\text { Midazolam, fentanyl, ondansetron, pantoprazole, } \\
\text { heparin }\end{array}$ & \\
\hline 44 & & $\begin{array}{l}\text { Midazolam, fentanyl, haloperidol, insulin, famotidine, } \\
\text { heparin }\end{array}$ & Methylprednisolone 125 mg every 6 hours $\times 2$ days \\
\hline 45 & & $\begin{array}{l}\text { Midazolam, fentanyl, ondansetron, insulin, } \\
\text { omeprazole, heparin }\end{array}$ & Hydrocortisone 100 mg every 8 hours $\times 3$ days \\
\hline 46 & $\begin{array}{l}\text { Linezolid, piperacillin/ } \\
\text { tazobactam, tobramycin }\end{array}$ & $\begin{array}{l}\text { Midazolam, Diltiazem, mycophenolate mofetil, } \\
\text { tacrolimus, famotidine, insulin, heparin }\end{array}$ & $\begin{array}{l}\text { Hydrocortisone } 100 \mathrm{mg} \text { every } 8 \text { hours } \times 6 \text { days, } \\
\text { prednisone } 40 \mathrm{mg} / \text { day } \times 3 \text { days }\end{array}$ \\
\hline 47 & $\begin{array}{l}\text { Vancomycin, cefipime, } \\
\text { levofloxacin }\end{array}$ & $\begin{array}{l}\text { Midazolam, aspirin, captopril, furosemide, simvastatin, } \\
\text { insulin }\end{array}$ & Prednisone $40 \mathrm{mg} /$ day $\times 3$ days, $30 \mathrm{mg} /$ day $\times 2$ days \\
\hline 49 & & $\begin{array}{l}\text { Midazolam, aspirin, atorvastatin, bisoprolol, } \\
\text { clopidogrel, fluticasone, folic acid, pantoprazole, } \\
\text { heparin }\end{array}$ & $\begin{array}{l}\text { Methylprednisolone } 60 \mathrm{mg} \text { every } 6 \text { hours } \times 4 \text { days, } \\
40 \mathrm{mg} \text { every } 12 \text { hours } \times 2 \text { days, prednisone } \\
40 \mathrm{mg} / \text { day } \times 1 \text { day }\end{array}$ \\
\hline 50 & $\begin{array}{l}\text { Vancomycin, aztreonam, } \\
\text { tobramycin, daptomycin }\end{array}$ & $\begin{array}{l}\text { Midazolam, hydroxyzine, darbepoetin, levothyroxine, } \\
\text { ferrous sulfate, ergocalciferol, pancrelipase, } \\
\text { omeprazole }\end{array}$ & Hydrocortisone 20 mg every 12 hours $\times 37$ days \\
\hline 51 & $\begin{array}{l}\text { Vancomycin, piperacillin/ } \\
\text { tazobactam, doripenam, } \\
\text { fluconazaole }\end{array}$ & $\begin{array}{l}\text { Midazolam, fentanyl, furosemide, pancrelipase, } \\
\text { magnesium oxide, famotidine, insulin, heparin }\end{array}$ & \\
\hline 52 & $\begin{array}{l}\text { Vancomycin, piperacillin/ } \\
\text { tazobactam, daptomycin }\end{array}$ & $\begin{array}{l}\text { Midazolam, fentanyl, levothyroxine, darbepoetin, } \\
\text { fluticasone, lactulose, paroxetime, insulin, heparin }\end{array}$ & Hydrocortisone 100 mg every 8 hours $\times 5$ days \\
\hline 53 & $\begin{array}{l}\text { Tamiflu, piperacillin/ } \\
\text { tazobactam, cefipime, } \\
\text { daptomycin, doripenam }\end{array}$ & $\begin{array}{l}\text { Midazolam, darbepoetin, bumetanide, famotidine, } \\
\text { insulin, heparin }\end{array}$ & \\
\hline 54 & $\begin{array}{l}\text { Vancomycin, piperacillin/ } \\
\text { tazobactam }\end{array}$ & $\begin{array}{l}\text { Morphine, oxycodone, furosemide, famotidine, insulin, } \\
\text { heparin }\end{array}$ & Hydrocortisone 50 mg every 6 hours $\times 3$ days \\
\hline 55 & $\begin{array}{l}\text { Vancomycin, piperacillin/ } \\
\text { tazobactam, levofloxacin, } \\
\text { daptomycin }\end{array}$ & $\begin{array}{l}\text { Ferrous sulfate, folic acid, levothyroxine, } \\
\text { metaclopramide, pravastatin, digoxin tacrolimus, } \\
\text { omeprazole, heparin }\end{array}$ & \\
\hline 56 & $\begin{array}{l}\text { Daptomycin, linezolid, } \\
\text { tobramycin, colistin, } \\
\text { zithromax }\end{array}$ & $\begin{array}{l}\text { Midazolam, fentanyl, dexmedetomidine, omeprazole, } \\
\text { heparin }\end{array}$ & \\
\hline 57 & $\begin{array}{l}\text { Vancomycin, piperacillin/ } \\
\text { tazobactam, levofloxacin, } \\
\text { tamiflu }\end{array}$ & $\begin{array}{l}\text { Midazolam, fentanyl, clonazepam, gabapentin, } \\
\text { famotidine, insulin, heparin }\end{array}$ & \\
\hline 58 & Piperacillin/tazobactam & $\begin{array}{l}\text { Midazolam, morphine, furosemide, metoprolol, } \\
\text { citalopram, pantoprazole, aspirin, acetazolamide, } \\
\text { insulin, heparin }\end{array}$ & $\begin{array}{l}\text { Methylprednisolone } 60 \mathrm{mg} \text { every } 8 \text { hours } \times 4 \text { days, } \\
\text { prednisone } 60 \mathrm{mg} / \text { day } \times 5 \text { days, } 40 \mathrm{mg} / \text { day } \times \\
5 \text { days, } 30 \mathrm{mg} / \text { day } \times 5 \text { days }\end{array}$ \\
\hline 59 & $\begin{array}{l}\text { Vancomycin, cefipime, } \\
\text { levofloxacin }\end{array}$ & $\begin{array}{l}\text { Midazolam, fentanyl, dopamine, norepinephrine, } \\
\text { simvastatin, aspirin, omeprazole, heparin }\end{array}$ & $\begin{array}{l}\text { Hydrocortisone } 100 \text { mg every } 8 \text { hours } \times 3 \text { days, } 50 \\
\text { mg every } 8 \text { hours } \times 2 \text { day, } 50 \text { mg every } 12 \text { hours } \times \\
7 \text { days }\end{array}$ \\
\hline 60 & $\begin{array}{l}\text { Vancomycin, cefipime, } \\
\text { tobramycin }\end{array}$ & $\begin{array}{l}\text { Midazolam, fentanyl, norepinephrine, pantoprazole, } \\
\text { heparin }\end{array}$ & \\
\hline
\end{tabular}


(A)

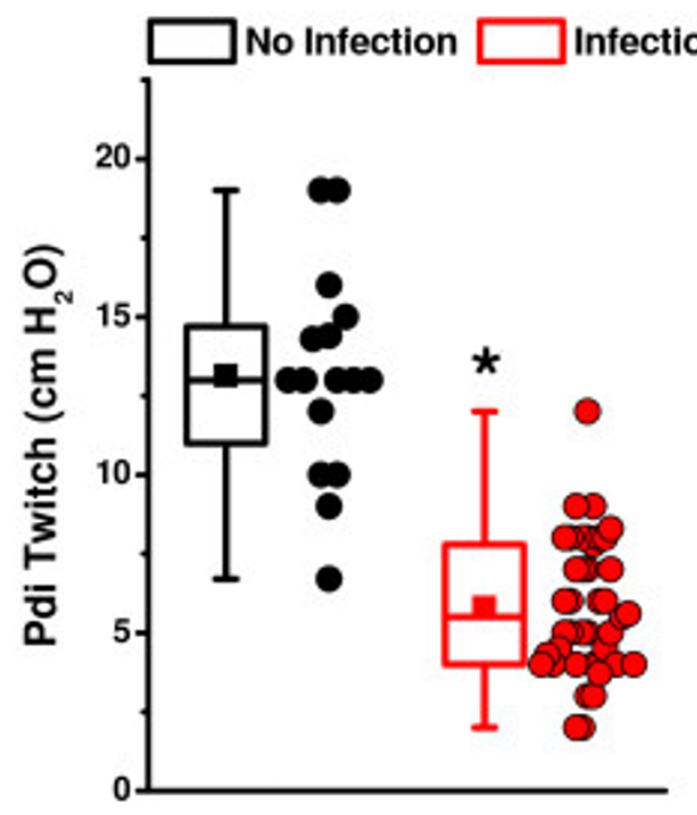

(B)

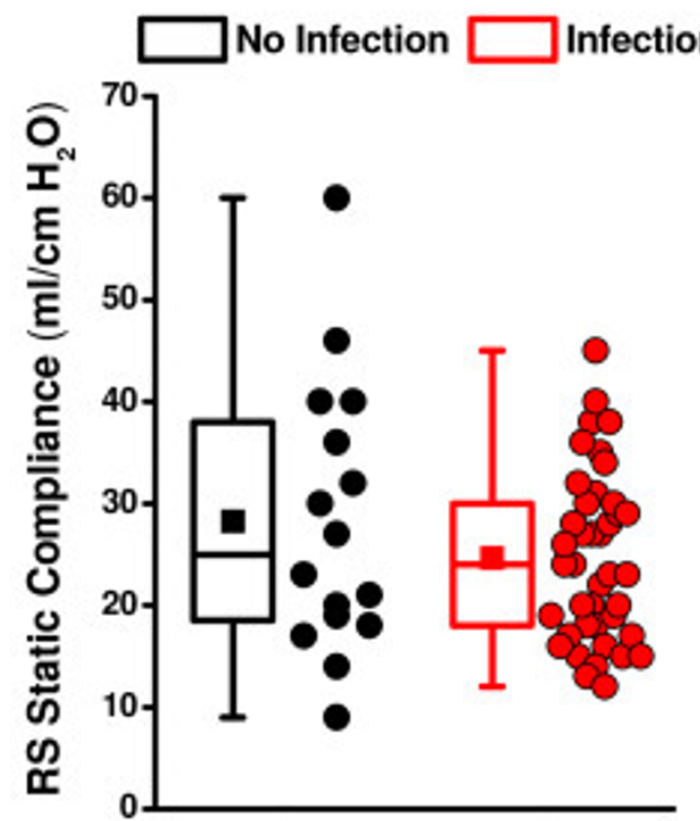

(C)

Figure 2 Infection and diaphragm weakness. (A) Transdiaphragmatic twitch pressure (PdiTw) measurements for non-infected and infected patients. Data from individual patients are shown for each group on the right, while plots on the left for each group show mean (filled squares), median levels (middle line of box), $25 \%$ and $75 \%$ confidence intervals (upper and lower borders of the box) and 1\% and 99\% intervals (whiskers above and below the box). Infection was associated with significant lower Pdi Twitch values (*statistical significance). (B) respiratory system (RS) static compliance and $(\mathbf{C})$ inspiratory airway resistance for non-infected and infected patients; there was no difference in these indices of lung function between non-infected and infected groups. 


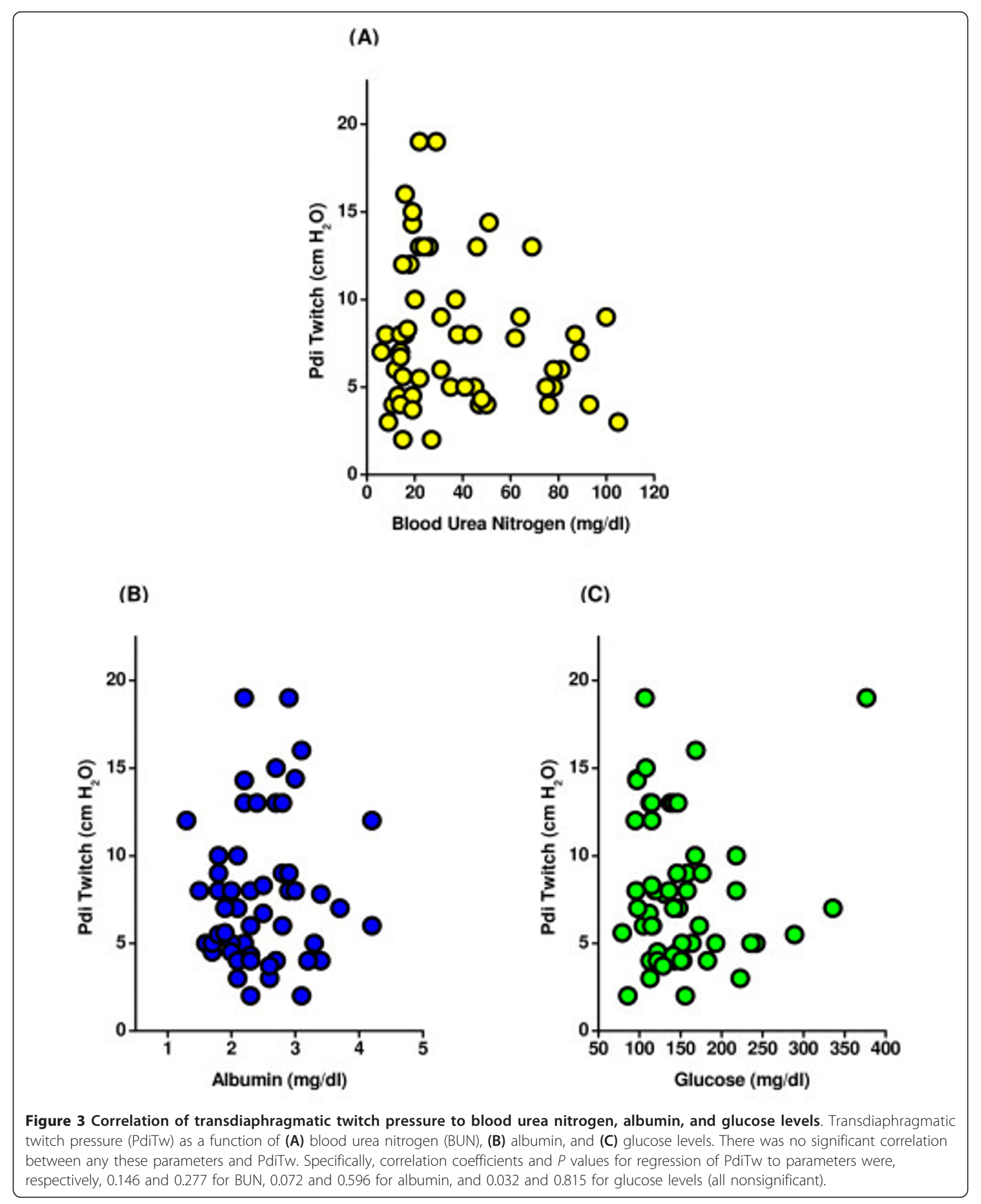


(A)

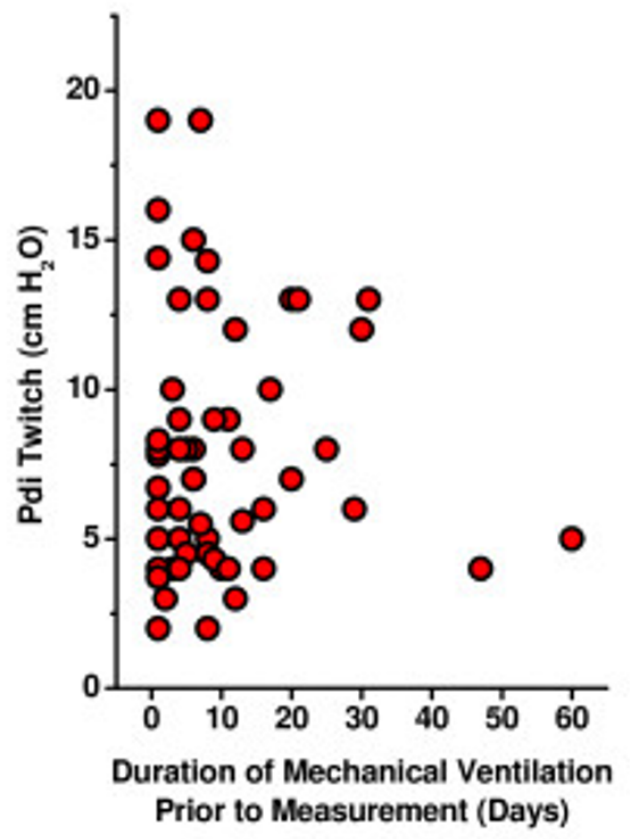

(B)

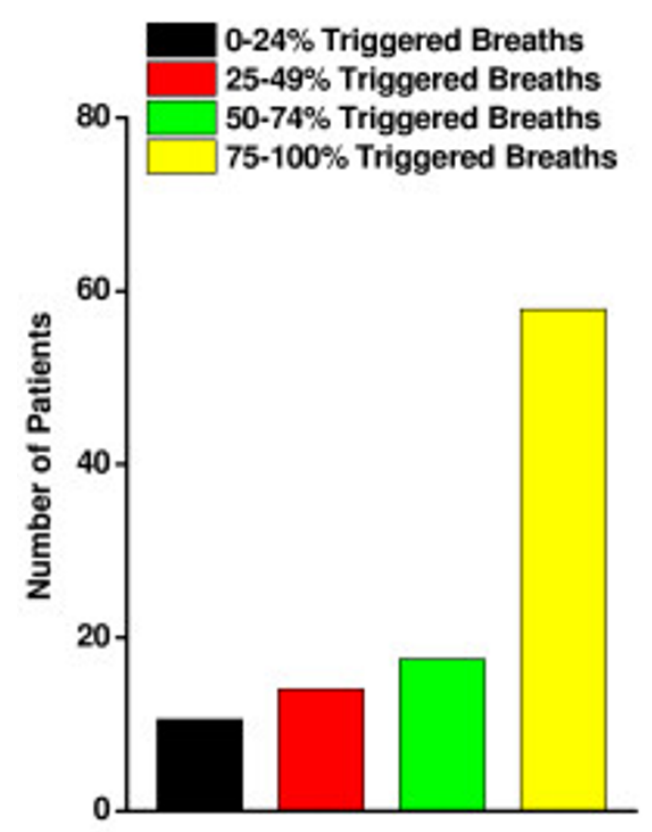

(C)

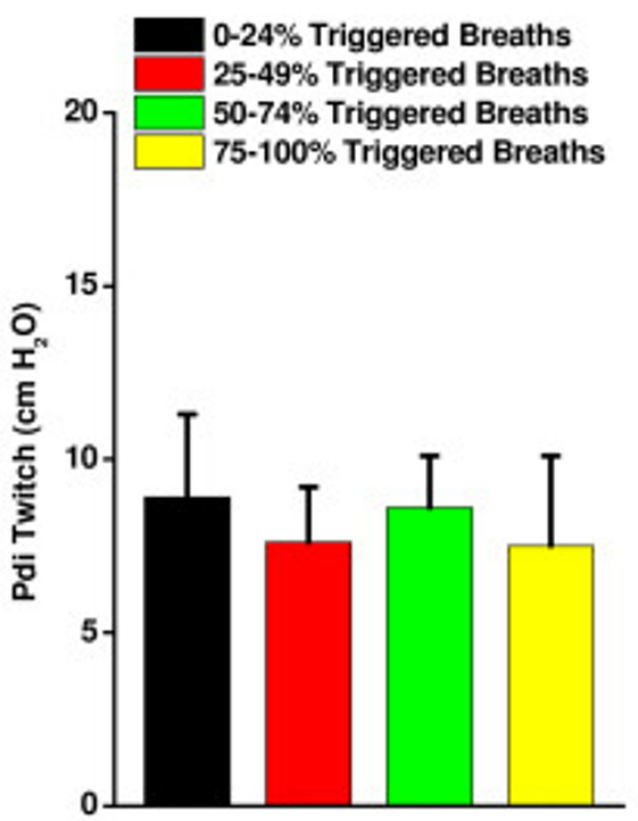

Figure 4 Relationship of prior duration of mechanical ventilation and ventilator triggering to diaphragm strength. (A) Transdiaphragmatic twitch pressure (PdiTw) as a function of the duration of mechanical ventilation prior to measurement of PdiTw. There was no statistically significant correlation of PdiTw to duration of ventilation prior to measurement, with a correlation coefficient of 0.020 and $P=0.881$ for this assessment (nonsignificant). (B) The majority of subjects actively initiated (that is, triggered) ventilator breaths more than $75 \%$ of the time. (C) The level of diaphragm strength (PdiTw) did not correlate with the level of triggering, with the same PdiTw observed at all triggering levels. 


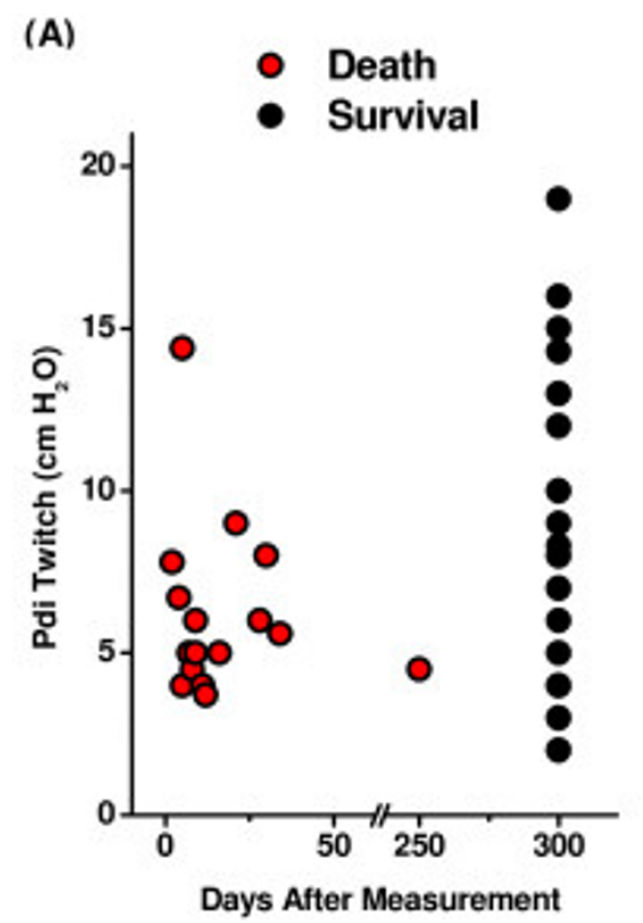

(C)

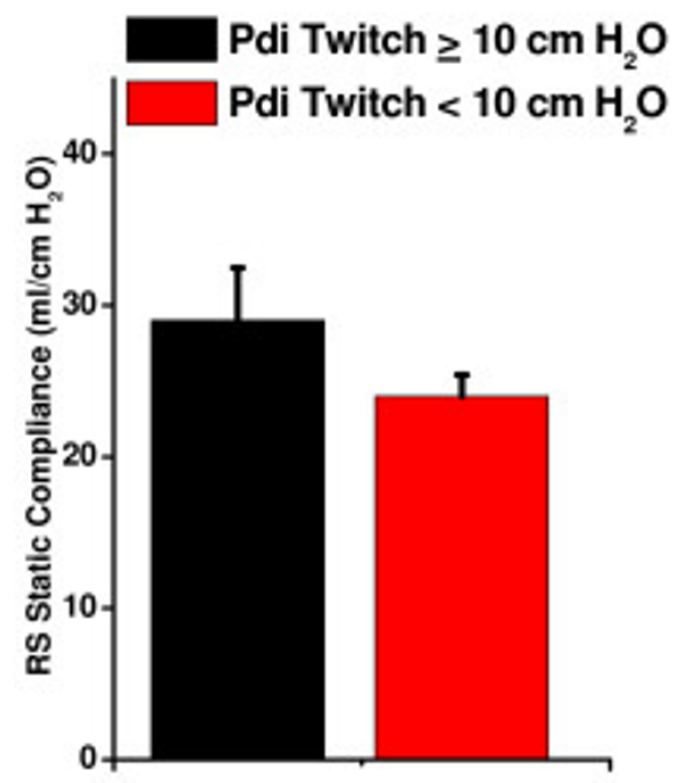

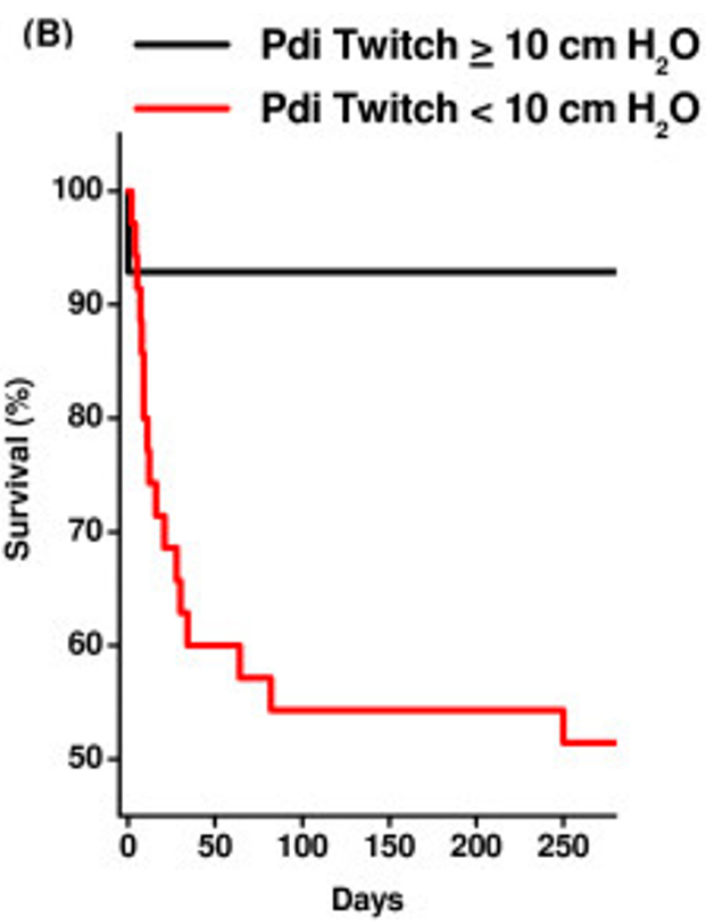

(D)

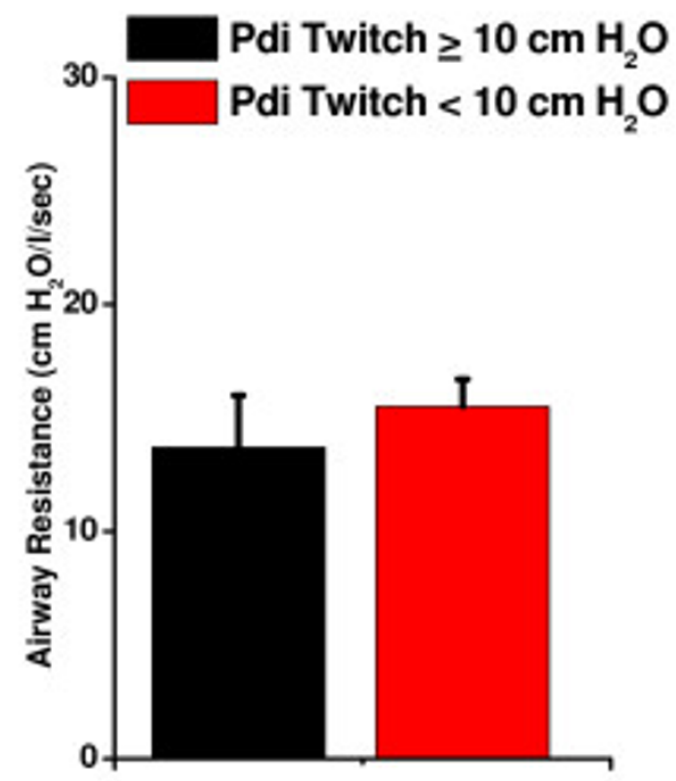

Figure 5 Relationship of diaphragm strength to survival. (A) Survival of patients (days after measurement, $x$ axis) as a function of transdiaphragmatic twitch pressure (PdiTw) level ( $y$ axis). Patients that died had low average PdiTw levels $\left(6.3 \pm 0.6 \mathrm{cmH}_{2} \mathrm{O}\right)$ while survivors had higher PdiTw levels $\left(8.9 \pm 0.9 \mathrm{cmH}_{2} \mathrm{O}, P=0.044\right)$. (B) Survival curves for subjects with PdiTw $\geq 10 \mathrm{cmH}_{2} \mathrm{O}(n=15)$ and PdiTw $<10 \mathrm{cmH} \mathrm{H}_{2} \mathrm{O}(n=$ 42). Weak subjects had a significantly higher mortality (49\%) than strong subjects ( $7 \%, P=0.022)$. To exclude the possibility that the greater mortality in the weakest patients may have been due to the presence of more severe lung dysfunction, we also examined (C) respiratory system (RS) static compliance and (D) airway resistance. There was no significant difference in RS static compliance or airway resistance for patients with PdiTw $\geq 10 \mathrm{cmH}_{2} \mathrm{O}$ and PdiTw $<10 \mathrm{cmH}_{2} \mathrm{O}$, indicating that the greater mortality ion the weakest patients was not due to concomitant lung dysfunction. 


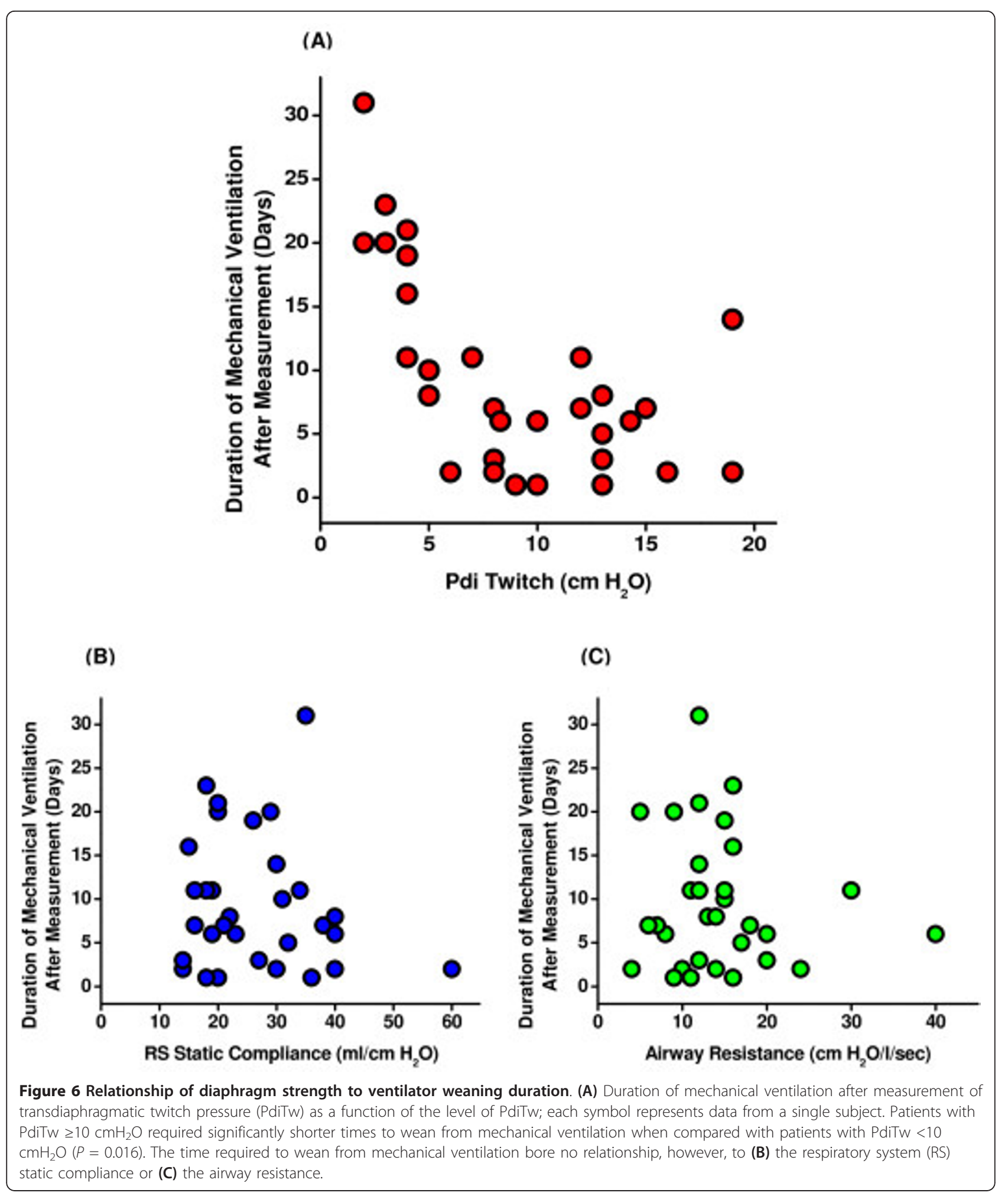

attempts. Weakness almost certainly contributed to the inability to wean these patients from mechanical ventilation and thereby may have influenced the decision to withdraw care.
We also found that a high percentage of patients with PdiTw $<10 \mathrm{cmH}_{2} \mathrm{O}$ required transfer to LTAC units. Reports indicate that long-term outcomes for this group of patients are poor, with a high percentage (51\%) dying 
within 1 year [19]. As a result, the high rate of transfer of weak patients to these units represents a poor outcome. In addition, we found that the relationship between diaphragm strength and duration of mechanical ventilation for patients that did not die and remained in the ICU was curvilinear, with duration increasing progressively as PdiTw levels fell to lower values (Figure 6A). Weak patients with PdiTw $<10 \mathrm{cmH}_{2} \mathrm{O}$ required more than twice as long to wean from mechanical ventilation than stronger patients with PdiTw levels $\geq 10 \mathrm{cmH}_{2} \mathrm{O}$. Moreover, the duration of mechanical ventilation did not correlate with the level of lung dysfunction but only with the level of diaphragm strength.

We also evaluated our data to ascertain the role of infections, BUN, albumin and glucose levels in the induction of diaphragm weakness in mechanically ventilated patients. We found the level of diaphragm weakness in mechanically ventilated MICU patients did not correlate with BUN, glucose, or albumin levels despite previous reports associating these factors with prolonged mechanical ventilation [11-13]. In contrast, we found that evidence of infection was a predictor of strikingly lower levels of diaphragm strength than that observed for noninfected patients. This finding is consistent with multiple previous animal studies demonstrating that infection rapidly reduces diaphragm force generation, decreases diaphragm mitochondrial function, activates diaphragm proteolytic pathways, and reduces diaphragm contractile protein function [9,20-26].

While infected patients had the weakest diaphragms, even the non-infected mechanically ventilated patients in our study had a median level of PdiTw $\left(13 \mathrm{cmH}_{2} \mathrm{O}\right)$, which is substantially lower than that observed for normal healthy adults $\left(30 \mathrm{cmH}_{2} \mathrm{O}\right)$. There are at least two potential explanations for the weakness observed in the non-infected patients. First, many of the patients in our study were chronically ill, with multiple comorbid conditions including heart failure, malignancy, liver and renal diseases. Each of these entities has negative effects on muscle function, and it is possible that the pre-intubation muscle function of these patients may have been appreciably lower than that observed in normal subjects.

In addition, use of mechanical ventilation can result in diaphragm inactivity and atrophy [27-30]. Numerous animal studies have provided evidence of this phenomenon, and, more recently, several elegant studies indicate that loss of diaphragm function occurs in patients who are subjected to controlled mechanical ventilation with minimal or no spontaneous respirations $[18,27]$. Our patients were all ventilated using assisted modes of mechanical ventilation and may therefore have had less inactivity-induced diaphragm dysfunction than observed in patient populations ventilated with controlled modes of mechanical ventilation. Nevertheless, it is still possible that ventilator-induced inactivity contributed to the level of diaphragm weakness observed in our non-infected patients. As a corollary, the level of weakness observed in our infected patients may represent the combined effects of chronic illness, ventilator-induced inactivity, and infection-induced diaphragm dysfunction.

One should note, however, that the non-infected patients had a level of PdiTw (median of $13 \mathrm{cmH}_{2} \mathrm{O}$ ) that was sufficiently high for this group of patients to be expected to have good outcomes (that is, a low death rate and an average wean time from mechanical ventilation of about 5 days) according to the data presented in Figures 5 and 6 . Only the infected mechanically ventilated patients, as a group, had low enough PdiTw levels (median of 5.5 $\mathrm{cmH}_{2} \mathrm{O}$ ) to expect poor outcomes (that is, a high mortality and a protracted need for mechanical ventilation). These data therefore argue that even if all of the diaphragm weakness observed in our non-infected patients was a consequence of ventilator-induced inactivity, this level of weakness alone would not be expected to result in poor patient outcomes. Our data would instead suggest that only the combination of ventilator-induced inactivity and infection may produce sufficient diaphragm weakness to negatively influence patient survival and duration of mechanical ventilation.

\section{Conclusions}

In summary, we found that mechanically ventilated MICU patients have severe diaphragm weakness, that the clinicians caring for these patients greatly underestimate the severity of diaphragm weakness present, and that infections are a major risk factor for the development of diaphragm weakness in this population. We also found that diaphragm weakness was associated with poor patient outcomes, including a significantly increased mortality, an increased transfer to LTACs and a markedly longer duration required for weaning from mechanical ventilation.

Diaphragm weakness appears to be a major risk factor for respiratory failure and death in mechanically ventilated MICU patients; theoretically, pharmacological treatments that improve diaphragm strength should reduce the duration of mechanical ventilation and MICU mortality. Currently no such agents are used in clinical practice, but recent experimental studies indicate that pharmacological inhibition of selected cellular pathways can prevent diaphragm weakness in animal models of critical illness [31,32]. There is an urgent need to translate these pharmacological treatments from the bench to the bedside in order to prevent or reverse diaphragm weakness in mechanically ventilated MICU patients. Such therapies are likely to influence both acute and long-term outcomes. 


\section{Key messages}

- Recent work indicates that many mechanically ventilated MICU patients have severe diaphragm weakness, but the causes and consequences of this weakness remain controversial.

- The present study indicates that infection is a major risk factor for development of diaphragm weakness in MICU patients treated with assist modes of mechanical ventilation.

- This work also demonstrates that the level of diaphragm weakness is a novel predictor of clinical outcomes; the weakest patients have a high mortality and require prolonged durations of mechanical ventilation. - This study indicates that clinicians underestimate the severity of diaphragm dysfunction in mechanically ventilated critically ill patients.

\section{Additional material}

\section{Additional file 1: presents additional methods}

Additional file 2: Table S1 presenting the demographic and clinical data in $\mathbf{5 7}$ subjects who underwent PdiTw measurements.

\section{Additional file 3: Figure S1. Relationship of PdiTw values to PEEP}

levels. PdiTw values are shown as a function of PEEP levels. We found no correlation between the level of PEEP and PdiTw measurements. The correlation coefficient and $p$ value for regression of PdiTw to PEEP were 0.093 and 0.492 , respectively (NS).

Additional file 4: Figure S2. Effect of Steroids on PdiTw values. Cumulative dosages of steroids was calculated and converted into hydrocortisone equivalents, then plotted as a function of PdiTw. As shown, steroid dosage had no relationship to measured PdiTw. The correlation coefficient and $p$ value for regression of PdiTw to hydrocortisone equivalents were 0.064 and 0.637 , respectively (NS).

\section{Abbreviations}

BUN: blood urea nitrogen; LTAC: long-term acute care; MICU: medical ICU; PdiTw: transdiaphragmatic twitch pressure; PEEP: positive end-expiratory pressure.

\section{Competing interests}

The authors declare that they have no competing interests.

\section{Authors' contributions}

GSS drafted the protocol, performed the measurements, analyzed the pressure tracings, obtained patient data, interpreted the data, and drafted and revised the final manuscript. LAC assisted in performing the measurements, obtaining patient data, and had a major impact on the interpretation of the data and revision of the manuscript. Both authors read and approved the final manuscript.

\section{Acknowledgements}

This work was supported by grants from the National Institutes of Health to GSS (HL 100239 and HL113494).

Received: 23 January 2013 Revised: 9 April 2013

Accepted: 20 June 2013 Published: 20 June 2013

\section{References}

1. Wunsch $H$, Linde-Zwirble WT, Angus DC, Hartman ME, Milbrandt EB, Kahn JM: The epidemiology of mechanical ventilation use in the United States. Crit Care Med 2010, 38:1947-1953.
2. Luhr OR, Karlsson M, Thorsteinsson A, Rylander C, Frostell CG: The impact of respiratory variables on mortality in non-ARDS and ARDS patients requiring mechanical ventilation. Intensive Care Med 2000, 26:508-517.

3. Dasta JF, McLaughlin TP, Mody SH, Piech CT: Daily cost of an intensive care unit day: the contribution of mechanical ventilation. Crit Care Med 2005, 33:1266-1271.

4. Laghi F, Cattapan SE, Jubran A, Parthasarathy S, Warshawsky P, Choi YS, Tobin MJ: Is weaning failure caused by low-frequency fatigue of the diaphragm? Am J Respir Crit Care Med 2003, 167:120-127.

5. Watson AC, Hughes PD, Louise Harris M, Hart N, Ware RJ, Wendon J, Green M, Moxham J: Measurement of twitch transdiaphragmatic, esophageal, and endotracheal tube pressure with bilateral anterolateral magnetic phrenic nerve stimulation in patients in the intensive care unit. Crit Care Med 2001, 29:1325-1331.

6. Hermans G, Agten A, Testelmans D, Decramer M, Gayan-Ramirez G: Increased duration of mechanical ventilation is associated with decreased diaphragmatic force: a prospective observational study. Crit Care 2010, 14:R127.

7. Jaber S, Jung B, Matecki S, Petrof BJ: Clinical review: ventilator-induced diaphragmatic dysfunction - human studies confirm animal model findings! Crit Care 2011, 15:206.

8. Petrof BJ, Jaber S, Matecki S: Ventilator-induced diaphragmatic dysfunction. Curr Opin Crit Care 2010, 16:19-25.

9. Callahan LA, Supinski GS: Sepsis-induced myopathy. Crit Care Med 2009, 37:S354-S367.

10. Barreiro E, Comtois AS, Mohammed S, Lands LC, Hussain SN: Role of heme oxygenases in sepsis-induced diaphragmatic contractile dysfunction and oxidative stress. Am J Physiol Lung Cell Mol Physiol 2002, 283:L476-L484.

11. Modawal A, Candadai NP, Mandell KM, Moore ES, Hornung RW, Ho ML, Tsevat J: Weaning success among ventilator-dependent patients in a rehabilitation facility. Arch Phys Med Rehabil 2002, 83:154-157.

12. Hermans G, Wilmer A, Meersseman W, Milants I, Wouters PJ, Bobbaers H, Bruyninckx F, Van den Berghe G: Impact of intensive insulin therapy on neuromuscular complications and ventilator dependency in the medical intensive care unit. Am J Respir Crit Care Med 2007, 175:480-489.

13. Wu YK, Kao KC, Hsu KH, Hsieh MJ, Tsai YH: Predictors of successful weaning from prolonged mechanical ventilation in Taiwan. Respir Med 2009, 103:1189-1195.

14. Luo YM, Hart N, Mustfa N, Man WD, Rafferty GF, Polkey MI, Moxham J: Reproducibility of twitch and sniff transdiaphragmatic pressures. Respir Physiol Neurobiol 2002, 132:301-306.

15. Topeli A, Laghi F, Tobin MJ: Can diaphragmatic contractility be assessed by twitch airway pressures in patients with chronic obstructive pulmonary disease? Am J Respir Crit Care Med 1999, 160:1369-1374.

16. Aubier M, Murciano D, Lecocguic $Y$, Viires $N$, Jacquens $Y$, Squara $P$, Pariente R: Effect of hypophosphatemia on diaphragmatic contractility in patients with acute respiratory failure. $N$ Engl I Med 1985, 313:420-424.

17. De Muth JE: Overview of biostatistics used in clinical research. Am J Health Syst Pharm 2009, 66:70-81.

18. Jaber S, Petrof BJ, Jung B, Chanques G, Berthet JP, Rabuel C, Bouyabrine H, Courouble P, Koechlin-Ramonatxo C, Sebbane M, Similowski T, Scheuermann V, Mebazaa A, Capdevila X, Mornet D, Mercier J, Lacampagne A, Philips A, Matecki S: Rapidly progressive diaphragmatic weakness and injury during mechanical ventilation in humans. Am J Respir Crit Care Med 2011, 183:364-371.

19. Schonhofer B, Euteneuer S, Nava S, Suchi S, Kohler D: Survival of mechanically ventilated patients admitted to a specialised weaning centre. Intensive Care Med 2002, 28:908-916.

20. Supinski GS, Wang W, Callahan LA: Caspase and calpain activation both contribute to sepsis-induced diaphragmatic weakness. J Appl Physiol 2009, 107:1389-1396.

21. Callahan LA, Supinski GS: Sepsis induces diaphragm electron transport chain dysfunction and protein depletion. Am J Respir Crit Care Med 2005, 172:861-868.

22. Callahan LA, Nethery D, Stofan D, DiMarco A, Supinski G: Free radicalinduced contractile protein dysfunction in endotoxin-induced sepsis. Am J Respir Cell Mol Biol 2001, 24:210-217.

23. van Hees HW, Schellekens WJ, Linkels M, Leenders F, Zoll J, Donders $R$, Dekhuijzen PN, van der Hoeven JG, Heunks LM: Plasma from septic shock patients induces loss of muscle protein. Crit Care 2011, 15:R233. 
24. Mofarrahi M, Sigala I, Guo Y, Godin R, Davis EC, Petrof B, Sandri M, Burelle $Y$, Hussain SN: Autophagy and skeletal muscles in sepsis. PLOS ONE 2012, 7: e47265.

25. Peruchi BB, Petronilho F, Rojas HA, Constantino L, Mina F, Vuolo F, Cardoso MR, Goncalves CL, Rezin GT, Streck EL, Dal-Pizzol F: Skeletal muscle electron transport chain dysfunction after sepsis in rats. J Surg Res 2011, 167:e333-e338.

26. Supinski GS, Callahan LA: Caspase activation contributes to endotoxininduced diaphragm weakness. J Appl Physiol 2006, 100:1770-1777.

27. Levine S, Nguyen T, Taylor N, Friscia ME, Budak MT, Rothenberg P, Zhu J, Sachdeva R, Sonnad S, Kaiser LR, Rubinstein NA, Powers SK, Shrager JB: Rapid disuse atrophy of diaphragm fibers in mechanically ventilated humans. N Engl J Med 2008, 358:1327-1335.

28. Hudson MB, Smuder AJ, Nelson WB, Bruells CS, Levine S, Powers SK: Both high level pressure support ventilation and controlled mechanical ventilation induce diaphragm dysfunction and atrophy. Crit Care Med 2012, 40:1254-1260.

29. Maes K, Testelmans D, Powers S, Decramer M, Gayan-Ramirez G: Leupeptin inhibits ventilator-induced diaphragm dysfunction in rats. Am J Respir Crit Care Med 2007, 175:1134-1138.

30. DeRuisseau KC, Kavazis AN, Deering MA, Falk DJ, Van Gammeren D, Yimlamai T, Ordway GA, Powers SK: Mechanical ventilation induces alterations of the ubiquitin-proteasome pathway in the diaphragm J Appl Physiol 2005, 98:1314-1321

31. Supinski GS, Vanags J, Callahan LA: Eicosapentaenoic acid preserves diaphragm force generation following endotoxin administration. Crit Care 2010, 14:R35.

32. Supinski GS, Callahan LA: Calpain activation contributes to endotoxininduced diaphragmatic dysfunction. Am J Respir Cell Mol Biol 2010, 42:80-87.

doi:10.1186/cc12792

Cite this article as: Supinski and Ann Callahan: Diaphragm weakness in mechanically ventilated critically ill patients. Critical Care 2013 17:R120.

\section{Submit your next manuscript to BioMed Central} and take full advantage of:

- Convenient online submission

- Thorough peer review

- No space constraints or color figure charges

- Immediate publication on acceptance

- Inclusion in PubMed, CAS, Scopus and Google Scholar

- Research which is freely available for redistribution

Submit your manuscript at www.biomedcentral.com/submit
Ciomed Central 